\title{
A Worthwhile Measurement of Early Vigilance and Therapeutic Monitor in Axial Spondyloarthritis: A Literature Review of Quantitative Sacroiliac Scintigraphy
}

\author{
Authors: \\ Zhu Wei Lim, ${ }^{1}$ Shih-Chuan Tsai,, ${ }^{2,3}$ Yi-Ching Lin, ${ }^{2,3}$ Yuan-Yang Cheng, ,,5,6 \\ *Shin-Tsu Chang, ${ }^{4,7,8}$ \\ 1. Department of Obstetrics and Gynecology, Changhua Christian Hospital, \\ Changhua, Taiwan \\ 2. Department of Nuclear Medicine, Taichung Veterans General Hospital, Taichung, \\ Taiwan \\ 3. Department of Medical Imaging and Radiological Science (Institute of Radiological \\ Science), Central Taiwan University of Science and Technology, Taichung, Taiwan \\ 4. Department of Physical Medicine and Rehabilitation, Taichung Veterans General \\ Hospital, Taichung, Taiwan \\ 5. Center for Geriatrics and Gerontology, Taichung Veterans General Hospital, \\ Taichung, Taiwan \\ 6. School of Medicine, Yangming Campus (Taipei), National Yang Ming Chiao Tung \\ University, Hsinchu, Taiwan \\ 7. Department of Physical Medicine and Rehabilitation, Kaohsiung Veterans General \\ Hospital, Kaohsiung, Taiwan \\ 8. Department of Physical Medicine and Rehabilitation, Tri-Service General Hospital, \\ School of Medicine, National Defense Medical Center, Taipei, Taiwan \\ *Correspondence to ccdivlaser1959@gmail.com
}

Disclosure:

The authors have declared no conflicts of interest. This review did not receive any specific grant from funding agencies in the public, commercial, or not-for-profit sectors.

Acknowledgements: The authors would like to thank Esther Yi Liu for her secretary assistance. Authors Lim and Chang conceived and designed the experiments. Authors Lim, Tsai, Lin, and Chang performed the experiments. Authors Lim, Cheng, and Chang analysed the data. Authors Tsai and Lin contributed reagents/materials and tools. Authors Lim and Tsai wrote the paper and authors contributed equally.

Received:

23.01.21

Accepted:

25.05.21

Keywords:

Anti-streptolysin O titre, bone scan, osteitis condensans ilii, periostitis, poststreptococcal reactive arthritis (PSRA), spondyloarthropathy (SpA).

Citation:

EMJ Rheumatol. 2021;8[1]:129-139.

\section{Abstract}

Background: Back pain a common cause for hospital visits. Nuclear skeletal scintigraphy, at a high sensitivity, provides a functional imaging for detecting bone diseases. Sacroiliitis is an inflammation of the sacroiliac joint. Bone scan with quantitative sacroiliac scintigraphy (QSS) has been a useful inflammation indicator for sacroiliac joints. However, QSS has been ignored in the rehabilitation practice. 
Objective: To present the background, mechanisms, and current clinical applications of bone scan with QSS in spondyloarthropathy (SpA).

Methods: The authors performed a literature review of QSS through database searching of MEDLINE, Embase, CINAHL, HaPI, Cochrane Review, and citation mining. Studies were included if they had QSS in the methodology performed in adult patients with various diseases. Any articles, including the authors', that can be performed in a clinical setting were enrolled. Articles explicitly referencing QSS were retained for screening.

Results: QSS appearance of SpA, including ankylosing spondylitis, may give rise to early detection. The specificity of sacroiliitis based on QSS increases from 73\% to 97\%. After investigating the relationship between serum C-reactive protein and sacroiliac joint inflammation in patients with SpA, there appeared to be a significant difference between serum C-reactive protein in serum and in sacroiliac ratio (particularly the middle part of the both joints), indicating a systemic inflammatory response to flair-up of SpA, for example, serum C-reactive protein as an indicator of inflammation. Sacroiliitis also occurs in post-streptococcal reactive arthritis. The involvement of sacroiliac joints in the development of post-streptococcal reactive arthritis had been demonstrated a significant correlation between antistreptolysin O titres and QSS in patients with post-streptococcal reactive arthritis. Lower extremity periostitis acts as a human model in the study of bottom-up processing for periostitis-induced sacroiliac pain. The use of QSS can also monitor sacroiliac joint dysfunction before and after laser therapy. Improvements of the sacroiliac joint after convalescing of foot periostitis have been reported.

Conclusions: Bone scan using QSS is a good screening measurement in scintigraphy rehabilitation for early detection of SpA and raises awareness of physicians toward the next step of diagnosis.

\section{INTRODUCTION}

Back pain is a common cause for hospital visits. The pain origins for these patients can be determined using various methods. MRI and nuclear skeletal scintigraphy are clinical tools to determine certain aetiologies contributing to back pain. MRI is the first choice for examination over the past four to five decades. MRI produces precision images of bone and soft tissues in 3D displays. MRI is, nevertheless, not a good diagnostic tool for bone surveying and the procedure is time-consuming, costly, and has contraindications, for example the presence of cerebral aneurysm clips, cardiac pacemakers, and cochlear implants. ${ }^{1}$ Breathing movements during MRI can lead to distorted images. ${ }^{2}$ MRI produces images of specific body regions, and poor cortical bone details with only $70 \%$ sensitivity in detecting areas of active inflammation prior to the development of structural lesions., ${ }^{1,3}$

In contrast to CT or MRI, nuclear skeletal scintigraphy provides functional imaging for bones of the entire body at a reasonable cost, as well as high sensitivity for various bone diseases despite of a lower resolution of the bone scan. Such bone scans are the most widely used method to diagnose bone diseases and being critical in monitoring bone metastases. ${ }^{4,5}$ This may be posed as, in analogy, CT/MRI sees individual trees but not the forest, and nuclear medicine sees the forest but not individual trees.

Seronegative spondyloarthrithis (SpA) is closely associated with back pain and its early diagnosis is crucial. Sacroilitis being the earlier symptom is, therefore, an important condition. Physicians, especially those of rheumatology and rehabilitation, typically diagnose SpA based on the international standards, like the Amor and Assessment of Spondyloarthritis International Society (ASAS) criteria. In the Amor criteria, 13 criteria are used to classify SpA with no image evidence. $^{6}$ The diagnosis of SpA has been modified in several versions from 1990 to $2016 .^{6-}$ ${ }^{16}$ Before the start of SpA treatment, sacroiliitis could be caused by other diseases like psoriatic arthritis, Reiter's syndrome, reactive arthritis, inflammatory bowel disease, or those that are inflammatory bowel disease related. These other causes need to be excluded first. ${ }^{17}$ Scientists have developed deep learning-based algorithms that can be use to detect sacroiliitis and grade 
the classification of SpA on plain radiograph with high sensitivity and accuracy.18,19 These approaches have overcome the interobserver variability in image interpretation. However, a group of researchers reported that technetium 99m-methyl diphosphonate (Tc-99m MDP) bone scan is more useful than plain radiographs in the early detection of SpA after study of 136 sacroiliac (SI) joints (42 patients and 26 controls) for 1 year and concluded that some patients $(n=2)$ with negative findings from plain radiograph and $\mathrm{MRI}$ showed positive results in a bone scan. ${ }^{20}$

Clinicians often accentuate on the uncomfortable or tender region pointed out by the patient. Nuclear skeletal scintigraphy is an alternative method to determine the exact location of discomfort. There are four common types of scans: whole body bone scan, ${ }^{21}$ three-phase bone scan, ${ }^{22}$ single photon emission CT (single photon emission CT [SPECT] $)^{23}$ and quantitative SI scintigraphy (QSS). ${ }^{24}$ These four scans all use the same radiotracer, Tc-99m MDP, which can be taken up by human skeleton. SPECT along or combined with CT as hybrid imaging can detect the exact location of neck pain in facet joint disorder, ${ }^{25}$ and raise the alarm of the sternoclavicular joint inflammation during flareup in psoriatic arthritis. ${ }^{26}$

Although other radiotracers, such as Tc-99mpyrophosphat, or Tc-99m hydroxymethylene diphosphonate, can be used instead, doctors of nuclear medicine still prefer Tc-99m-MDP as the choice of radiological compound. ${ }^{27} \mathrm{Tc}-99 \mathrm{~m}$ MDP is typically delivered in the bloodstream by an intravenous injection to disseminate in the body before being deposited in the bone. The primary mechanism of detecting bone lesions based on Tc-99m MDP is an abnormal accumulation due to osteogenic activity on the bone surface secondary to the calcium uptake in the affected area. Nuclear medicine bone scans can also present as a photopenic or cold zone in some bone lesions, such as the condition of bone necrosis, severe bone damage-like trauma, or tumour cell invasions leading to ischaemic changes. ${ }^{28,29}$

In this paper, the authors reviewed an alternative strategy: nuclear medicine bone scan with QSS to approach a patient with a comprehensive check-up in rehabilitation practice.

\section{METHODS}

To examine clinical application of QSS, the authors developed a search strategy of QSS using a literature review through database of MEDLINE, Embase (Ovid), CINAHL (EBSCOhost), HaPI (Ovid), Cochrane Review (Ovid), and citation mining. The research was not limited to articles published in English because abstracts in English were available. Studies were included if those had QSS in methodology performed in adult patients with various diseases. Any articles, including the authors', that can be performed in a clinical setting were enrolled. Articles explicitly referencing QSS were retained for screening. The searches were conducted on $3^{\text {rd }}$ May 2017 and updated on $31^{\text {st }}$ March 2021. Summarised here are the most relevant aspects of the studies of SpA and different diagnoses of SpA according to different clinical imaging, and a critical discussion on the potential advantages and disadvantages of QSS.

\section{RESULTS}

\section{Quantitative Sacroiliac Scintigraphy Is an Easier Way to Approach Sacroiliitis Compared to Unspecified Physical Examination}

Sacroiliitis is an inflammation of the SI joint as the result of systemic diseases or stress (e.g. abnormal shearing force). The inflammation in the $\mathrm{SI}$ joint is also seen in individuals without $\mathrm{SpA}$, including postpartum females, recreational runners, military recruits, professional ice hockey players, and healthy controls with or without symptomatic back pain. ${ }^{30-33}$ A substantial portion of those people displayed sacroiliitis and bone marrow oedema on MRI at baseline. ${ }^{31,32,34,35}$ Those structural lesions without the development of fat metaplasia have shown a more mechanical than inflammatory origin. ${ }^{34}$ In addition, the CT Syndesmophyte Score (CTSS), SI joints scores, and MRI lesions were not significantly increased in those people after a period of time, which indicated that stress may also cause sacroiliitis. ${ }^{31,33,34,36}$

Clinical manifestations of sacroiliitis are lower back pain, inguinal ligament pain, or buttocks pain, and even radiated pain to hamstrings similar to sciatica. ${ }^{37}$ 

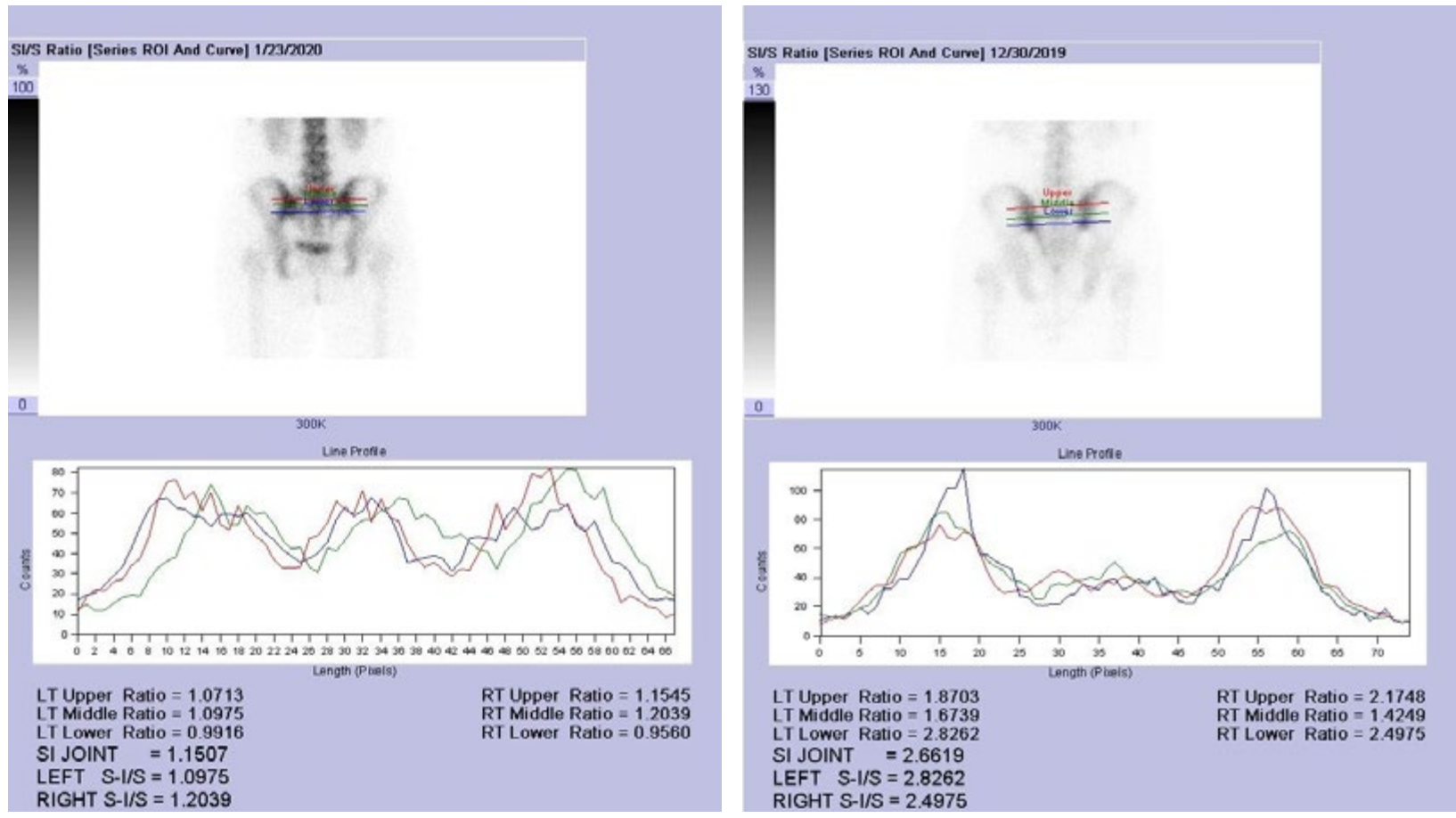

Figure 1: Methods used to obtain a quantitative sacroiliac joint-to-sacrum ratio.

From the ROI based on accountable images, the ratio is obtained after measuring total number of counts within the region of $\mathrm{SI}$ joint and divided by total number of counts within an equal-size region at the sacrum. The ratio for the upper (red), middle (green), and lower (blue) parts of both joints can be measured individually.

Left panel: a subject with normal SI joint. Right panel: a patient with AS.

AS: ankylosing spondylitis; ROI: region of interest; SI/S: quantitative sacroiliac joint-to-sacrum ratio.

The Fortin finger test may indicate the area potentially generating the pain by asking patient to point at the area of pain to the clinician. ${ }^{38}$ Those patients who fail to point out their painful areas would be identified to have poor outcome of their disease. Patrick's test is another method of physical examination for sacroiliitis. In this method, the examiner stretches the patient's SI joints according to the following motions: flexion, abduction, and external rotation of the hip joint. ${ }^{39} \mathrm{~A}$ positive test result is one in which refers to back pain or buttock pain, whereas groin pain is a sign that is more indicative of sacroiliitis. ${ }^{39}$ In addition, Gaenslen's sign is a test of sacroiliitis by abducting one side of hip joint and extending the other side in counter-rotation. ${ }^{40}$ The result is same as in the Patrick's test. ${ }^{40}$ Another test for sacroiliitis is the posterior superior iliac spines (PSIS) distraction test. ${ }^{41}$ A positive result is feeling pain when a medial-to-lateral direction force is applied on PSIS. ${ }^{41}$ In brief, there are many methods to test for sacroiliitis, but all with low specificity. ${ }^{42}$
Nuclear medicine bone scan with QSS has been a useful inflammation indicator for $\mathrm{SI}$ joint for the last 50 years. QSS is typically done following an intravenous injection of about $750 \mathrm{MBq}$ Tc-99m MDP. Planar imaging of the spine and SI joints achieves in the antero-posterior projections 3-4 hours after the injection, using a gamma camera via an elliptical course $\left(360^{\circ}, 64\right.$ projections, 20 $\mathrm{sec} /$ projection) the camera acquires images around the SI joints, storing in $128 \times 128$ matrices. Using the region-of-interest (ROI) method, a quantitative SI joint-to-sacrum ratio (or SI ratio), is computed based on counts at similar regions measured at the SI joint and at the sacrum. ${ }^{43}$ The ratio for the upper, middle, and lower parts of both joints is measured individually (Figure 1). In brief, the inflammation is considered as negative if the ratio is $<1.3$, or equivocal if around 1.4 , or positive if $>1.5$.

In 1977, Buell et al. ${ }^{44}$ were the first to report higher $\mathrm{SI}$ ratios in patients with $\mathrm{SI}$ disease. In 1998, Kaçar et al. ${ }^{45}$ reported that for subjects 
20 to 60 years old, the normal ranges of counts of $\mathrm{SI}$ joint versus over counts of sacrum ROI were between 0.74 and 1.22 for females, and between 0.87 and 1.31 for males. The reference $\mathrm{SI}$ ratios were estimated to be an average of $<1.3200$ for normal subjects and $<1.3812$ for late arthritic patients, while even higher at 1.52002.0900 for those with early arthritis. It was reported that patients with radiographic Grade I-II were considered to have early arthritis and patients with radiographic Grade III-IV were considered to have late arthritis. ${ }^{45}$ Tiwari et al. ${ }^{46}$ later showed four quantification methods of the SI joint index: irregular ROI, rectangular $\mathrm{ROI}$, profile peak counts, and profile-integrated counts. All of these methods gave similar results. Sebastjanowicz et al. ${ }^{47}$ reported a range of $\mathrm{SI}$ ratio between 1.18 and 2.28 for control subjects, with the highest standard deviation in paediatric patients. Therefore, SI ratio is a good measure for detecting early but not late arthritis. ${ }^{45}$ The maximum and minimum of $\mathrm{SI}$ values need to be considered for younger ( $<20$ years old) and older patients (>61 years old). ${ }^{47} \mathrm{SI}$ ratios can be measured individually as the upper, middle, and lower third due to distinct anatomic structures and kinetic physiology in the three parts of the joint on the bilateral sides. ${ }^{48}$

Unlike adults, ${ }^{49,50}$ children's quantitative SI indices may give good results using $L 5$ as the background ${ }^{51}$ or with the use of MRI because these younger subjects have more synovial enhancement without bone marrow oedema. ${ }^{52}$

The earlier study regarding QSS in healthy people was performed in a medical centre where a posterior planar film of the pelvis was obtained 3 hours after injection of 740 MBq 99mTc-MDP and the SI ratio was calculated. ${ }^{53}$ The results showed that the age-related changes in SI ratio are significant between sexes in certain age groups, but not lateralisation, and the SI ratios dropped steadily with age in females, whereas two plateaus appeared at ages 21 to 40, and 41 to 70 years in males. ${ }^{53}$

\section{DISCUSSION}

\section{Quantitative Sacroiliac Scintigraphy for Spondyloarthropathy, Including Ankylosing Spondylitis}

Ankylosing spondylitis (AS) is a disease of axial SpA but is often recorded as suspicious by radiologists after a plain X-ray, and is, therefore, sometimes diagnosed late. In the 1980 s, SI ratio was being used as important indicator in early vigilance of $A S$, particularly in males to prevent its progression to sacroiliitis. ${ }^{49,54-57}$ Although QSS showed a limited sensitivity and specificity of approximately $50 \%$ for each when appropriate controls were used, ${ }^{58,59}$ the SI ratio of 1.55 meant that AS disease progression was expected to last $<3$ years and $>3$ years at 1.40 .60

In 1993, Collie et al. ${ }^{61}$ measured SI ratios for six of 11 patients ( 5 female, 6 male), and found elevated ratios ( $>1.5$ for both $\mathrm{SI}$ joints) in four of them. After 2 years, two of these patients showed higher $\mathrm{SI}$ ratios that preceded plain roentgenologic abnormalities. In two patients, unilateral sacroiliitis on plain radiographs was confirmed as bilateral on QSS. They suggested that the scintigraphic appearance of AS, though not unique to the disease, offers the opportunity for early detection and vigilance, avoiding further unnecessary investigation and treatment for both suspected as well as unsuspecting cases. ${ }^{61}$

In addition, Koc et al. ${ }^{62}$ reported that with SI ratios, the specificity of sacroiliitis based on bone scans increases from $73 \%$ to $97 \%$ and negative predictions increase up to $91 \%$, in parallel with positive predictions. The authors of this current study concluded that with regard to time and cost, bone scan is slightly better than MRI and SPECT/CT in detecting AS and sacroiliitis. ${ }^{62}$

Elevation serum levels of high sensitivity C-reactive protein (hs-CRP) are considered a risk factor/biomarker for various diseases, including SpA. A previous study had retrospectively investigated the relationship between serum hs-CRP and SI joint inflammation in 29 patients with SpA ( $n=29$; mean age: 32.27 years; femalemale ratio: $6: 23$ ). All patients underwent hs-CRP testing and skeletal scintigraphic scans with QSS between January 2007 and September 2013. The results showed a significant difference between hs-CRP in serum and in SI ratio (particularly 
the middle part of $\mathrm{SI}$ joint, on both sides). It was concluded that the significantly high concentrations of serum hs-CRP may indicate a systemic inflammatory response to a flair-up of the SI joint and should be an indicator of $\mathrm{SI}$ inflammation in SpA. ${ }^{63}$

Although QSS may not be mandatory for most patients with suspected AS, an empirical practice has depicted that it has a role in selected cases where there is a very visible disorder in the absence of obvious roentgenologic changes For accurate diagnoses of SpA, QSS is only recommended when combined with a CT scan. ${ }^{64}$

The importance of concomitant use of CT in the assessment of $\mathrm{SI}$ joints along with scintigraphy includes the following: the combination of semi-quantitative analysis of CT and quantitative analysis of $\mathrm{SI}$ joints can increase the unique specification of the risk level for active sacroiliitis; 65 CT is the gold standard for bone erosion and superior to conventional radiography and $M R I, 65$ it enables the crosssectional, multi-planar visualisation of the pathologic processes, which was better than conventional radiography, ${ }^{66-68}$ in addition, the Modified New York Criteria scoring system for sacroiliitis can also applied to CT; by using spectral CT, fat deposition and bone marrow oedema can be measured similarly to MRI, which can increase the sensitivity for early changes of sacroiliitis; ${ }^{65,68}$ and, other than bony erosion and relative water and calcium ratio of the SI joint, CT can detect sclerosis and syndesmophytes, which could be helpful to identify differential diagnoses in chronic changes in sacroiliitis. ${ }^{65,68}$

\section{Quantitative Sacroiliac Scintigraphy for Post-Streptococcal Reactive Arthritis}

Titers of anti-streptolysin $O$ (ASLO) are of diagnostic value in the early detection of poststreptococcal reactive arthritis (PSRA), ${ }^{48,69-71}$ early arthritis after rheumatic fever, ${ }^{72-74}$ and movement disorder. ${ }^{75}$ The dividing line is normal if the titer is $\leq 116 \mathrm{IU} / \mathrm{mL}$, and abnormal if the titer is $>116 \mathrm{IU} / \mathrm{mL}$. PSRA is a non-suppurative sequela of a prior streptococcal infection.

In Asia, some scholars have demonstrated the involvement of the SI joint in the development of PSRA. In a study, a total of 84 subjects (mean age: 23.0; range: 18.0-36.4) underwent QSS and their ASLO titers were measured (range: 25-520 IU/
$\mathrm{mL}$ ). The SI joint was divided into three regions: upper, middle, and lower parts. Depending on fibrous cartilage, ligament, and the direction of the SI joint, bilateral QSS measurements of the three parts were collected. A highly significant correlation was found between ASLO titers and $S I$ ratios $(p<0.0001)$. An increment of $1 \mathrm{lU} / \mathrm{mL}$ of the titer resulted in a significant increment of SI ratio by 0.0008 units. It was also found that with the increased ASLO titer per unit, SI ratio increased significantly by 0.0008 units and thereafter 0.0074 units per additional year. ${ }^{48}$ The findings suggested that $\mathrm{SI}$ joint involvement is a manifestation of PSRA. The results demonstrated a strong correlation between the ASLO titer and the QSS in patients with PSRA. Subjects with SI joint involvement should be advised to have an ASLO titer measured and a QSS done. ${ }^{48}$

It was noticed that those patient with $\mathrm{SI}$ also reported upright postural abnormality. It was, therefore hypothesised that an imbalance of the lumbopelvis due to $\mathrm{SI}$ disorder might produce a positional change, and there would be different postural sway when standing upright. All subjects underwent 10 sway tests to assess static sway in an upright standing posture. With eyes open and plantar flexion, the high ASLO group had bigger values in sway area, sway velocity, and sway intensity. The values of sway velocity and intensity obtained with eyes open and plantar flexion and dorsiflexion had lower intensity values when compared with those obtained in closed eyes and plantar flexion and dorsiflexion in the high ASLO group, but not in the low ASLO group. Significant differences were found between the two groups in all sway values under all the tested position conditions. It is speculated that subjects with high levels of streptococcal serology have greater sways on all postural parameters compared to those with low serology. The speculation is consistent with proprioceptive deficits in the SI joint contributing to postural impairments, ${ }^{76,77}$ as shown in Figure 2. The use of QSS is the first of its kind to detect active SI joint disorder in the studies of PSRA.

\section{Quantitative Sacroiliac Scintigraphy for Osteitis Condensans Ilii}

Osteitis condensans ilii $(\mathrm{OCl})$, first reported in 1926, has symptoms that include axial lower back pain and premature arthritis. 


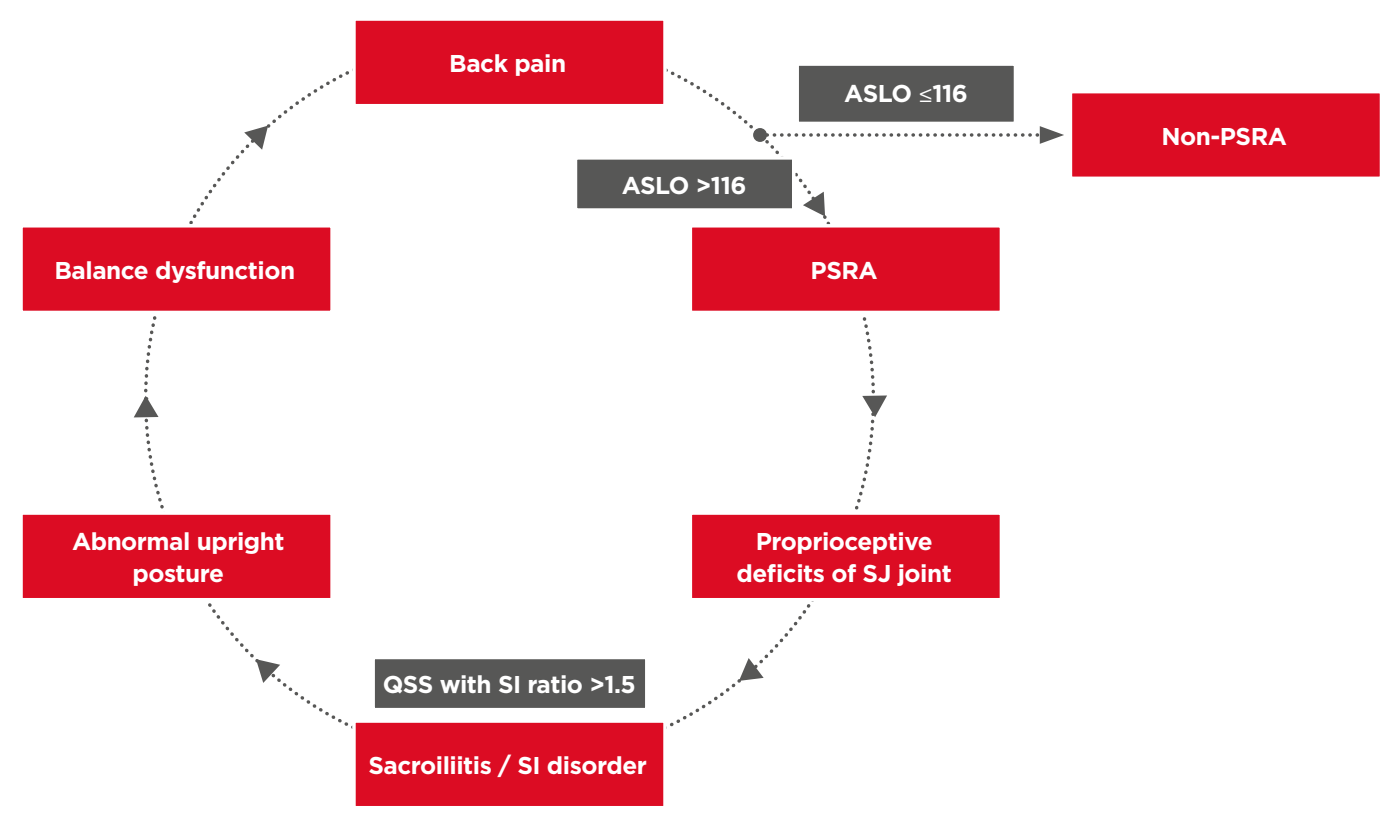

Figure 2: Schematic illustration of the pathophysiology of post-streptococcal reactive arthritis and its related disorders.

ASLO: anti-streptolysin O; PSRA: post-streptococcal reactive arthritis; QSS: quantitative sacroiliac scintigraphy; SI: sacroiliac.

It primarily affects females younger than the age of 40 , and often after pregnancy. Parperis $K$ et al. have suggested the term idiopathic pelvis sclerosis might be an alternative term to $\mathrm{OCl}$ due to the lack of inflammation evidence in those patients. ${ }^{78} \mathrm{OCl}$ is considered a diagnosis of exclusion because plain radiograph and MRI findings of the spine were unremarkable; ${ }^{79}$ while the characteristic X-ray image may show triangular sclerotic changes in the auricular portion of the bilateral ilium with preserved SI joint space. ${ }^{80,81}$ Patients mostly exhibit normal physical and neurological examination but some show SI joint region tenderness and increased lumbar lordosis. ${ }^{59}$ From a case-control study, $\mathrm{OCl}$ is shown to be associated with pain on SI stress tests. The preserved SI joint space is the key characteristic of $\mathrm{OCl}^{79}$ It has been postulated that $\mathrm{OCl}$ induces stress on the $\mathrm{SI}$ joint, leading to a piriformis muscle syndrome with sciatica. The association between $\mathrm{OCl}$ and $\mathrm{SI}$ joint stress, and that between sacroiliitis and sciatica, has been reported. It is characterised by a favourable prognosis; ${ }^{78}$ therefore, the treatment goal was to improve their health-related quality of life. ${ }^{78}$ In clinical practice, symptoms of $\mathrm{OCl}$ presented with piriformis muscle syndrome and sciatica could be successfully managed with SI joint injections. ${ }^{82}$ The use of QSS is the first of its kind to monitor active SI joint disorder before and after the treatment of $\mathrm{OCI}$. MRI, SPECT, and PET/ CT might have the ability to distinguish $\mathrm{OCl}$ from other diseases and require larger-scale studies before use in the clinical setting..$^{81,83,84}$

\section{Quantitative Sacroiliac Scintigraphy for Periostitis-Induced Sacroiliac Pain}

Juvenile-onset

HLA-B27-associated

'unclassifiable' SpA, especially cases without evidence of enteric or genitourinary symptoms, may have tarsal periostitis as an early clinical manifestation and then show bilateral sacroiliac pain later on. ${ }^{85}$ One article has reported improvements of the SI joint after convalesce of the foot periostitis, and concluded that stress events, exercise, and abnormal posture can all increase SI ratios, while corrected posture for a period of time, anti-inflammatory drugs, and rehabilitation programmes can all reduce SI ratios. ${ }^{86}$

Periostitis of the lower limbs is a common disorder from sports injuries. Foot periostitis is considered a human model in the study 
of bottom-up processing. Calin et al. ${ }^{87}$ have demonstrated that pelvic radiography showing fluffy periostitis was equally distributed among symptomatic, asymptomatic HLA-B27-positive, and symptomatic HLA-B27-negative control groups. ${ }^{87}$ The diagnosis of foot periostitis, therefore, can be confirmed by medical history and physical examination, as well as triple-phase bone scan using skeletal nuclear scintigraphy. One clinical study ${ }^{88,89}(n=54)$ explored functional improvements of the lower limbs after low-level laser therapy (LLLT) with regard to balance function, including postural stability testing and limits of stability. After therapy, there were significant improvements in pain score and balance dysfunction. The study concluded that LLLT is effective for treating lower-limb periostitis and even in short-duration interventions, and LLLT exerted a positive effect on proprioception in these patients. ${ }^{88,89}$

Furthermore, foot pain was hypothesised to induce defective biomechanics and might cause SI joint stress and convalesce of the foot periostitis could restore the abnormal SI joints. The results showed scintigraphic improvements in the SI ratio, indicating significant therapeutic effects on foot periostitis. There was also a significant association between the middle and lower parts of the SI joint. SI ratios for the middle part on both sides were significantly higher (0.06 units) compared to the lower part. In conclusion, the patients with $\mathrm{SI}$ joint stress, as the result of bottom-up processing of foot periostitis, could be treated successfully after convalesce of the foot periostitis by either LLLT or conventional treatments. ${ }^{86}$ In another report regarding medial tibial stress syndrome (MTSS), similar findings were also reported. Both MTSS and SI joint stress can be confirmed by nuclear scintigraphy. ${ }^{90}$

Outcome measures included the Lower Extremity Functional Scale (LEFS) and QSS. The results showed that after therapy, LEFS was significantly higher (38.45, $\mathrm{p}<0.0001)$, and QSS was significantly lower $(p<0.0001)$. There is also a significant association between the middle and lower parts of the SI joint. It has been confirmed that $\mathrm{SI}$ joint stress due to bottom-up processing of MTSS can be normalised after successful therapy of MTSS by LLLT. ${ }^{90}$ The use of QSS is the first of its kind to monitor SI joint dysfunction before and after the studies of periostitisinduced sacroiliac pain.

\section{Limitations of Quantitative Sacroiliac Scintigraphy}

QSS has several limitations. The first limitation is lower specificity in detecting chronic inflammation. SI values the in acute phase of stress-induced inflammation fall between 1.7 and 1.8 but nearly normal values tend to be reached after 6 months of recovery. The hyperfixation method for the SI joints may be used in treating chronic SI arthritis. The second limitation is related to the small overlaps in SI indices between lower back pain and sacroiliitis patients and controls. ${ }^{91}$ Therefore, the reference values and the cut-off values of SI joints need to be clearly established. ${ }^{38}$ In addition to this, stratification of age ${ }^{27,53,91}$ and sex $^{45,53}$ are needed to distinguish the overlapping area resulted from sacroiliitis. One literature review has included studies about well-defined AS populations only, suspected sacroiliitis, and/or mechanical lower back pain. ${ }^{92}$ They excluded the studies without preforming the SI joint-sacrum ratio or giving a clear definition of reference value of the SI jointsacrum ratio in quantitative scintigraphy and calculating sensitivity or specificity value..$^{92}$ They have concluded that although scintigraphy was nearly $50 \%$ cheaper and generally more available than MRI, the likelihood ratio of scintigraphy for diagnostic acute sacroiliitis was only between 2.5 and 3.0.92

The authors of the review suggested that QSS was limited for diagnostic values for AS diagnosis. ${ }^{92}$ Using auxiliary laboratory data and clinical scoring, including serum hs-CRP, vitamin $D$ levels, erythrocyte sedimentation rate, and Bath Ankylosing Spondylitis Disease Activity Index (BASDAI), clinicians may able to detect SI inflammation-related diseases from healthy controls. $^{93}$ A current article has suggested there is value of QSS in helping the differential diagnoses of back pain-associated conditions, such as suspected fracture, ${ }^{64}$ particularly in survivors with $\mathrm{SI}$ joint pain after experiencing a vehicle accident.

\section{CONCLUSION}

In this article, the authors have revisited skeletal scintigraphy using QSS on patients with SI joint dysfunction, who also present with myalgia, arthritic, or lower back pain. More 
than one underlying disorder may contribute to symptoms in these patients. Clinicians need to better understand the pathophysiological mechanisms and the origin of sacroiliitis before prescribing treatments. QSS is helpful in clarifying inflammation of $\mathrm{SpA}$ and in disease assessment. In conclusion, the skillful pattern recognition skills of the diagnosis of axial SpA are more important than overdiagnoses by a positive MRI finding. Bone scan using QSS is a good screening and follow-up measurement in scintigraphy rehabilitation for early detection and vigilance of SpA and raises awareness for physicians to adopt the SI ratio in the next steps towards diagnosis.

\section{References}

1. Shellock F et al. MR procedures and biomedical implants, materials, and devices: 1993 update. Radiology. 1993;189(2):587-99.

2. Khoo VS et al. Magnetic resonance imaging (MRI): considerations and applications in radiotherapy treatment planning. Radiother Oncol. 1997;42(1):1-15.

3. Zilber $\mathrm{K}$ et al. Radionuclide methods in the diagnosis of sacroiliitis in patients with spondyloarthritis: an update. Rambam Maimonides Med J. 2016;7(4).

4. Rybak L, Rosenthal D. Radiological imaging for the diagnosis of bone metastases. Q J Nucl Med. 2001;45(1):53-64.

5. Tombal B, Lecouvet F. Modern detection of prostate cancer's bone metastasis: is the bone scan era over? Adv Urol. 2011;2012:893193.

6. Amor B et al. Criteria of the classification of spondylarthropathies. Rev Rhum Mal Osteoartic. 1990;57(2):85-9. (In French).

7. Dougados $M$ et al. The European Spondylarthropathy Study Group preliminary criteria for the classification of spondylarthropathy. Arthritis Rheum. 1991;34(10):1218-27.

8. Rudwaleit $M$ et al. The development of assessment of spondyloarthritis international society classification criteria for axial spondyloarthritis (part ii): validation and final selection. Ann Rheum Dis. 2009;68(6):777-83.

9. Sieper $\mathrm{J}$ et al. New criteria for inflammatory back pain in patients with chronic back pain: a real patient exercise by experts from the Assessment of SpondyloArthritis international Society (ASAS). Ann Rheum Dise. 2009;68(6):784-8.

10. Rudwaleit M et al. The assessment of spondyloarthritis international society classification criteria for peripheral spondyloarthritis and for spondyloarthritis in general. Ann Rheum Dis. 2011;70(1):25-31.

11. Moltó A et al. Performances of the assessment of spondyloarthritis international society axial spondyloarthritis criteria for diagnostic and classification purposes in patients visiting a rheumatologist because of chronic back pain: results from a multicenter, cross $\bigotimes$ sectional study. Arthritis Care Res (Hoboken). 2013;65(9):1472-81.

12. van den Berg $R$ et al. ASAS modification of the Berlin algorithm for diagnosing axial spondyloarthritis: results from the SPondyloArthritis Caught Early (SPACE)-cohort and from the Assessment of SpondyloArthritis international Society (ASAS)-cohort. Ann Rheum Dis. 2013;72(10):1646-53.

13. Moltó $\mathrm{A}$ et al. Evaluation of the validity of the different arms of the ASAS set of criteria for axial spondyloarthritis and description of the different imaging abnormalities suggestive of spondyloarthritis: data from the DESIR cohort. Ann Rheum Dis. 2015;74(4):746-51.

14. Akkoc N, Khan MA. Looking into the new ASAS classification criteria for axial spondyloarthritis through the other side of the glass. Curr Rheum Rep. 2015;17(6):515.

15. Rudwaleit M. Spondyloarthropathies: Identifying axial SpA in young adults with chronic back pain. Nat Rev Rheum. 2016;12(7):378-80.

16. Sepriano A et al. Predictive validity of the ASAS classification criteria for axial and peripheral spondyloarthritis after follow-up in the ASAS cohort: a final analysis. Ann Rheum Dis. 2016;75(6):1034-42.

17. Kobak S et al. The prevalence of sacroiliitis and spondyloarthritis in patients with sarcoidosis. Int Rheumatol. 2014;2014:289454.

18. Grauhan NF et al. Towards accurate detection of axial spondyloarthritis by using deep learning to capture sacroiliac joints on plain radiographs. Research Square. 2021;DOI:10.21203/ rs.3.rs-379664/v1.

19. Noh $\mathrm{SH}$ et al. Automated detection of sacroiliitis on plain radiograph using EfficientDet algorithm in young patients with back pain: a pilot study. Research Square. 2021;DOI:10.21203/ rs.3.rs-272234/v1.

20. Shinto AS et al. Utility of $99 m$ Tc-MDP bone scan in comparison to MRI and plain radiographs for the diagnosis of early spondyloarthropathy. J Spinal Surg. 2014;1(1):12-5.

21. Fernandez-Ulloa $M$ et al. Orthopaedic nuclear medicine: the pelvis and hip. Semin Nucl Med. 1998;28:25-40.

22. Riccabona G. Nuclear medicine in diagnosis and therapy of bone and joint diseases. Nucl Med Rev Cent East Eur. 1999;2(1):42-52.

23. Sarikaya I et al. The role of single photon emission computed tomography in bone imaging. Semin Nucl Med. 2001;31:3-16.

24. Rosenthall L. Nuclear medicine techniques in arthritis. Rheum Dis Clin North Am. 1991;17(3):585-97.

25. Chang ST et al. Single-photon emission computed tomography/ computed tomography (hybrid imaging) in the diagnosis of unilateral facet joint arthritis after internal fixation for atlas fracture. HSOA J Med Study Res. 2019;2:010.

26. Chang ST, Tsai TW. Abrupt swollen bump of the shoulder girdle joint as the first presentation of flare-up in a case of axial spondyloarthritis. Can J Biomed Res Tech. 2020;3(5):1-5.

27. Lin WY, Wang SJ. The influence of two bone agents (99Tcmpyrophosphate and 99Tcmmethylenediphosphonate) on quantitative sacroiliac joint scintigraphy. Nucl Med Commun. 1996;17(12):1035-38.

28. Russell A, Lentle B, Percy J. Investigation of sacroiliac disease: Comparative evaluation of radiological and radionuclide techniques. J Rheumatol. 1984;11(5):578-79.

29. Russell AS et al. Investigation of sacroiliac disease: comparative evaluation of radiological and radionuclide techniques. J Rheumatol. 1975;2(1):45-51.

30. Weber U, Jurik AG, Zejden A, et al. Frequency and anatomic distribution of magnetic resonance imaging features in the sacroiliac joints of young athletes: exploring "background noise" toward a data driven definition of sacroiliitis in early spondyloarthritis. Arthritis 
Rheumatol. 2018;70(5):736-45.

31. Varkas $\mathrm{G}$ et al. Effect of mechanical stress on magnetic resonance imaging of the sacroiliac joints: assessment of military recruits by magnetic resonance imaging study. Rheumatology. 2018;57(3):508-13.

32. de Winter $\mathrm{J}$ et al. Magnetic resonance imaging of the Sacroiliac joints indicating sacroiliitis according to the assessment of spondyloarthritis International Society definition in healthy individuals, runners, and women with postpartum back pain. Arthritis Rheumatol. 2018;70(7):104248.

33. Barnsley L, Paiva J, Barnsley L. Frequency of pertinent MRI abnormalities of the sacroiliac joints of patients without spondyloarthropathies: a systematic review of the literature. Skeletal Radiol. 2021;s00256-021-03719-6.

34. Eshed I et al. Peripartum changes of the sacroiliac joints on MRI: increasing mechanical load correlating with signs of edema and inflammation kindling spondyloarthropathy in the genetically prone. Clin Rheumatol. 2015;34(8):1419-26.

35. Seven $\mathrm{S}$ et al. MRI lesions in the sacroiliac joints for differentiation of patients with axial spondyloarthritis from postpartum women, patients with disc herniation, cleaning staff, long distance runners and healthy persons-a prospective cross-sectional study of 204 participants. Arthritis Rheumatol. 2019;71(12):2034-46.

36. Lee KA et al. Computed tomographybased assessment of radiographic progression in spine and sacroiliac joints after pregnancy in women with ankylosing spondylitis. Research Square. 2021. [Epub ahead of print]

37. Visser $L$ et al. Sciatica-like symptoms and the sacroiliac joint: clinical features and differential diagnosis. Eur Spine J. 2013;22(7):1657-64.

38. Fortin JD, Falco FJ. The Fortin finger test: an indicator of sacroiliac pain. Am J Ortho (Belle Mead NJ). 1997;26:477-80

39. Ozgocmen $\mathrm{S}$ et al. The value of sacroiliac pain provocation tests in early active sacroiliitis. Clin Rheumatol. 2008;27(10):1275-82

40. Neustadt DH. Ankylosing spondylitis. Postgrad Med. 1977;61(1):124-35.

41. Kok HK et al. Imaging the patient with sacroiliac pain. Can Assoc Radiol J. 2016;67(1):41-51.

42. Werner CM et al. Distraction test of the posterior superior iliac spine (PSIS) in the diagnosis of sacroiliac joint arthropathy. BMC Surgery. 2013;13(1):52

43. Davis MC et al. Quantitative sacroiliac scintigraphy. The effect of method of selection of region of interest. Clin Nucl Med. 1984;9(6):334-40.
44. Buell U. The scintigraphic investigation of sacroiliac disease: influence of age on the uptake ratio between sacroiliac and os sacrum. J Nucl Med. 1977;18(12):1241-2.

45. Kaçar $\mathrm{G}$ et al. Quantitative sacroiliac joint scintigraphy in normal subjects and patients with sacroiliitis. Ann Nucl Med. 1998:12(3):169-73.

46. Tiwari BP, Basu S. Estimation of sacroiliac joint index in normal subjects of various age groups: comparative evaluation of four different methods of quantification in skeletal scintigraphy. Nucl Med Rev Cent East Eur. 2013;16(1):26-30

47. Sebastjanowicz $P$ et al. The reference of normal values of the sacroiliac joint index in bone scintigraphy. Pomeranian J Life Sci. 2016;62(2):525. (In Polish).

48. Chang ST et al. Evidence-based correlation between anti-streptolysin o serum titer and sacroiliac joint disorder. J Rheumatol. 2007;34(8):1746-52.

49. Schörner W, Haubold U. Methodological problems of sacroiliac joint scintigraphy (author's transl). Nuklearmedizin. 1981;20(5):241-6. (In German).

50. Pitkänen $M$ et al. Quantitative sacroiliac scintigraphy: I. Methodological aspects. Scand J Rheumatol. 1982;11(4):199-202.

51. Bozkurt MF, Kiratli P. Quantitative sacroiliac scintigraphy for pediatric patients: comparison of two methods. Ann Nuclear Med. 2014;28(3):227-31.

52. Lin C et al. Magnetic resonance imaging findings in juvenile spondyloarthropathy and effects of treatment observed on subsequent imaging. Pediatr Rheumatol Online J. 2014;12(1):1-8.

53. Lin WY, Wang SJ. Influence of age and gender on quantitative sacroiliac joint scintigraphy. J Nucl Med. 1998;39(7):1269-72.

54. Domeij-Nyberg B et al. The reliability of quantitative bone scanning in sacroiliitis. Scand J Rheumatol. 1980;9(2):77-9

55. Pfannenstiel $P$ et al. Comparative study of quantitating 99m Tc-EHDP uptake in sacroiliac scintigraphy. Eur J Nucl Med. 1980;5(1):49-55.

56. Chase WF et al. The clinical usefulness of radionuclide scintigraphy in suspected sacroiliitis: A prospective study. $\mathrm{Br} J$ Rheumatol. 1983;22(2):67-72.

57. Lowe J et al. Significance of bone scintigraphy in symptomatic spondylolysis. Spine (Phila Pa 1976) 1984;9(6):653-5.

58. Miron SD et al. The value of quantitative sacroiliac scintigraphy in detection of sacroiliitis. Clin Rheumatol. 1983;2(4):407-14.
59. Lemmens AJ et al. Radiological and scintigraphic findings in patients with a clinical history of chronic inflammatory back pain. Skeleta Radiol. 1985;14(4):243-8.

60. Fam AG et al. Computed tomography in the diagnosis of early ankylosing spondylitis. Arthritis Rheum. 1985;28(8):930-37.

61. Collie D et al. 99mTc-MDP scintigraphy in ankylosing spondylitis. Clin Radiol. 1993;48(6):392-97.

62. Koc ZP et al. Sacroiliac indicis increase the specificity of bone scintigraphy in the diagnosis of sacroiliitis. Mol Imaging Radionucl Ther. 2015;24(1):8-14.

63. Liu TJ et al. Relationship of HS CRP and sacroiliac joint inflammation in undifferentiated spondyloarthritis. Open Med (Wars). 2018;13(1):113-8.

64. Wei JC et al. Clinical practice recommendations for the use of imaging in the diagnosis and management of axial spondyloarthritis in Taiwan. Int J Rheum Dis. 2020;23(1):24-36

65. Ran $\mathrm{J}$ et al. Role for imaging in spondyloarthritis. Quar J Nucl Med Mol Imaging. 2017;61(3):271-282.

66. Slobodin $\mathrm{G}$ et al. Incidental computed tomography sacroiliitis: clinical significance and inappropriateness of the New York radiological grading criteria for the diagnosis. Clin Rheumatol. 2012;31(3):425-8.

67. Lv $\mathrm{P}$ et al. Differentiation of smal hepatic hemangioma from small hepatocellular carcinoma: recently introduced spectral CT method. Radiology. 2011;259(3):720-9.

68. Zhang $P$ et al. A novel diagnostic method (spectral computed tomography of sacroiliac joints) for axial spondyloarthritis. J Formos Med Assoc. 2016;115(8):658-64.

69. Mackie S, Keat A. Poststreptococcal reactive arthritis: what is it and how do we know? Rheumatology (Oxford). 2004;43(8):949-54.

70. Sarakbi HA et al. Poststreptococcal reactive arthritis and the association with tendonitis, tenosynovitis, and enthesitis. J Clin Rheumatol. 2010;16(1):3-6.

71. Uziel $Y$ et al. Post-streptococcal reactive arthritis in children: a distinct entity from acute rheumatic fever. Pediatr Rheumatol Online. 2011;9(1):32

72. Visser $\mathrm{H}$ et al. The diagnostic value of streptococcal serology in early arthritis: a prospective cohort study. Rheumatology (Oxford). 2000;39(12):1351-6.

73. Ayoub EM et al. Group A streptococcal antibodies in subjects with or without rheumatic fever in areas with high or low incidences of rheumatic fever. Clin Diagn Lab 
Immunol. 2003:10(5):886-90.

74. Kotby AA et al. Antistreptolysin O titer in health and disease: levels and significance. Pediatr Rep. 2012;4(1):25-29.

75. Martino D, Giovannoni G. Autoaggressive immune-mediated movement disorders. Adv Neurol. 2005;96:320-35.

76. Chang CC et al. Impairment of static upright posture in subjects with undifferentiated arthritis in sacroiliac joint in conjunction with elevation of streptococcal serology. J Back Musculoskelet Rehabil. 2009;22(1):3341

77. Lu SC et al. Orthostatic postural deficit in subjects with streptococcal serology-related unclassified arthritis of the lumbopelvis. Tw J Phys Med Rehabil 2010;38(1):1-9. (In Chinese).

78. Parperis $\mathrm{K}$ et al. Osteitis condensans ilii: current knowledge and diagnostic approach. Rheumatol Int. 2020;40(7):1013-9.

79. Jenks $\mathrm{K}$ et al. Osteitis condensans ilii: a significant association with sacroiliac joint tenderness in women. Int J Rheum Dis. 2009;12(1):39-43.

80. Mitra R. Osteitis condensans ilii.
Rheumatol Int. 2010;30(3):293.

81. Gemmel F et al. SPECT/CT of osteitis condensans ilii: one-stop shop imaging. Clin Nucl Med. 2011;36(1):5961.

82. Chang ST et al. Relief of sciatica after sacroiliac joint injection in a female with osteitis condensans ilii. Asia Life Sci. 2016;25(1):515-9

83. Clarke DP et al. Magnetic resonance imaging of osteitis condensans ilii. $\mathrm{Br}$ J Rheumatol. 1994;33(6):599-600.

84. Lee YC et al. FDG uptake in sacroiliac joint due to osteitis condensans ilii shown on PET/CT in a patient with breast cancer: the value of coregistered CT in avoiding misinterpretation. Clin Nucl Med. 2012;37(5):e121-3.

85. Khan MA, van der Linden SM. A wider spectrum of spondyloarthropathies. Semin Arthritis Rheum 1990;20(2):107-13.

86. Chang CC et al. Improvement of sacroiliac joint stress bottom-up after convalesce of foot periostitis: a randomized controlled trial. Asia Life Sci. 2016;25(1):137-49.

87. Calin A et al. The close correlation between symptoms and disease expression in HLA B27 positive individuals. J Rheumatol.

1977;4(3):277-81.

88. Su HY et al. Effects of low power laser on pain and balance dysfunction caused by periostitis of lower limb: a case series. Tw J Phys Med Rehabil 2013; 41(1):51-7. (In Chinese).

89. Chang CC et al. Five-day, low-leve laser therapy for sports-related lower extremity periostitis in adult men: a randomized, controlled trial. Lasers Med Sci. 2014;29(4):1485-94.

90. Chen CL et al. Randomized controlled trial of laser therapy versus conventional therapy in the treatment of medial tibial stress syndrome. Int J Orthop Rehabil. 2016; 3(2):43-50.

91. Verlooy $\mathrm{H}$ et al. Quantitative scintigraphy of the sacroiliac joints. Clin imaging. 1992;16(4):230-3.

92. Song I et al. The diagnostic value of scintigraphy in assessing sacroiliitis in ankylosing spondylitis: a systematic literature research. Ann Rheum Dis. 2008;67(11):1535-40.

93. Pokhai GG et al. Vitamin D levels in ankylosing spondylitis: does deficiency correspond to disease activity? Rev Bras Reumatol. 2014;54(4):330-4 